\title{
AN EXTENSION OF THE SEARCH FOR SPECTROSCOPIC BINARIES IN M3 ${ }^{1}$
}

\author{
Carlton P. Pryor \\ Department of Physics, University of Victoria \\ Victoria, British Columbia V8W 2Y2, Canada \\ David W. Latham and Martha L. Hazen-Liller \\ Harvard-Smithsonian Center for Astrophysics \\ 60 Garden Street \\ Cambridge, Massachusetts 02138, USA
}

\begin{abstract}
We have obtained 295 new radial velocities for the 112 giants in the globular cluster M3 previously observed by Gunn and Griffin. Our velocities have a typical accuracy of $0.8 \mathrm{~km} / \mathrm{s}$ per measurement and have been combined with the Gunn and Griffin data in order to search for radial velocity variations over a time span of ten years. We find no convincing evidence that any of the giants observed are spectroscopic binaries with one notable exception, von Zeipel 164, which we believe is the first spectroscopic binary to be found in a globular cluster. Modelling of the velocity variations that would be expected in our data for a variety of binary populations confirms Gunn and Griffin's conclusion that binaries with separations of less than 10 AU must occur much less frequently among the giants of M3 than among the population I field stars.
\end{abstract}

\section{INTRODUCTION}

In 1980 we began programs to measure accurate radial velocities of faint stars in order to study the dynamics of clusters and the frequency of binaries in a variety of environments. Our basic approach was to obtain digital spectra with photon-counting Reticon detectors and echelle spectrographs on the telescopes at Mt. Hopkins. These spectra were then cross-correlated against a set of standard template spectra using modified versions of the analysis software originally developed for the CfA Redshift Survey (Tonry and Davis 1979).

In 1979 Gunn and Griffin (hereafter GG) published their excellent velocities for 111 giants in the globular cluster M3, and we undertook to remeasure several of their stars with the 1.5-m Tillinghast Reflector. Our initial results proved to have the same accuracies as the GG data. Moreover, no obvious velocity variables emerged when the two data sets were combined, further supporting GG's claim that globular clusters are deficient in binaries. Subsequently we undertook a major program to reobserve all of GG's objects

lobservations reported here were obtained in part with the Multiple Mirror Telescope, a joint facility of the Smithsonian and the University of Arizona. 
one or more times, using the Multiple Mirror Telescope (MMT) as well as the $1.5-\mathrm{m}$ telescope. In section 2 we summarize the radial velocity observations for the handful of giants in M3 which appear to have variable velocities.

Our data, when combined with that of GG, should allow the detection of most of the binaries with periods less than 10 years (their velocity amplitudes would exceed $15 \mathrm{~km} / \mathrm{s}$ peak-to-peak for two 0.8 solar mass stars in circular orbits viewed edge-on) and should also provide some constraints on the frequency of binaries with longer periods. We have used a two-parameter model of the binary population (one parameter governs the overall fraction of the stars which occur in binaries while the other sets the lower cutoff to the allowed binary separations), and we have determined the parameter ranges that are not excluded by the data. The results of this modelling are summarized in section 3.

\section{VELOCITY VARIABLES}

We have found only one giant in M3, von Zeipel (vZ) 164, that shows good evidence for velocity variations due to orbital motion. The four observations covering ten years are listed in Table I. The total amplitude is at least $18 \mathrm{~km} / \mathrm{s}$ and the period appears to be a few years. Note that $v Z 164$ is more than twenty core radii away from the cluster core, and we are fairly confident that the correct star was observed each time, because the field is not at all crowded. We believe that $v Z 164$ is a spectroscopic binary, the first to be found in a globular cluster.

TABLE I. Radial velocities for $v Z 164$, a giant in M3 that appears to be a spectroscopic binary.

\begin{tabular}{llll}
\hline Velocity & Error & Julian Day & Telescope \\
\hline-141.21 & & 2442199.70 & Palomar \\
-159.78 & 0.7 & 2445477.74 & MMT \\
-151.38 & 1.2 & 2445842.83 & 1.5 meter \\
-151.69 & 1.3 & 2445867.74 & 1.5 meter \\
\hline
\end{tabular}

There are two classes of mechanisms that may have removed primordial binaries from the sample of giant stars that we observed in M3. For long period, widely separated, and weakly bound systems, encounters with other stars tend to disrupt the binary. Binaries with orbital velocities larger than the cluster velocity dispersion ( $9 \mathrm{~km} / \mathrm{s}$ at the center) have a good chance of surviving. For systems with circular orbits and one solar mass total, this corresponds to a separation of roughly $10 \mathrm{AU}$. On the other hand, primordial binaries that are too close will be modified or even disrupted by mechanisms such as mass loss or mass transfer during the evolution of the primary from a main sequence star to a giant. The giants in $\mathrm{M} 3$ have radii as large as $0.4 \mathrm{AU}$, but it is hard to see how mass loss or mass transfer could be effective at modifying binaries with separations larger than about 1 AU. Thus there should be a window of separations between roughly 1 and $10 \mathrm{AU}$ where primordial binaries in M3 could survive, corresponding to a velocity window of roughly 30 to $9 \mathrm{~km} / \mathrm{s}$ for systems with circular orbits and one solar mass total. It may be 
significant that the first spectroscopic binary to be found among the giants in M3 appears to be in this velocity window and is located in the outer region of the cluster, where stellar encounters are much rarer than in the cluster center.

GG found that two stars, $v Z 764$ and 911 , fell off the cluster mean velocity of $-146.9 \mathrm{~km} / \mathrm{s}$ by +3.5 and -4.5 times the central velocity dispersion of the cluster (if the dispersion is calculated with the two high-velocity stars rejected). We observed these two stars several times, and we find no evidence for any velocity variation.

In addition to the two well-known pulsating variables $v Z 318$ and 803, our velocity data also provide evidence for small, seemingly random, velocity variations in some of the bightest cluster giants. The total range of variation can be as high as $5 \mathrm{~km} / \mathrm{s}$. We find six stars, each with at least five observations, for which the chi-square values of the differences have a probability of occurrence of less than 1 percent: vZ 490, 807, 858, 1053, 1397, and 1449. All six of these stars are among the brightest in the cluster, but only half the stars brighter than photovisual magnitude 12.8 appear to have this variability. Mayor et al. (1984) have recently reported the independent discovery of a similar phenomenon in 47 Tuc.

\section{BINARY FREQUENCY}

Harris and McClure (1983) have claimed that GG's failure to detect spectroscopic binaries among the giants in globular clusters is not inconsistent with the fairly high frequency of binaries that they found in a survey of field giants. They argued that the large diameters of the globular cluster giants observed by GG would remove short-period binaries with large orbital velocities from the sample. In addition, the smaller masses of the cluster giants would also reduce the velocity amplitudes for any binaries that might survive, compared to the younger field giants. We have taken advantage of the larger data set available to us (111 stars with multiple observations compared to GG's 33, and a time coverage of ten years compared to their four) and have modelled the residuals that would be expected in our data for a two-parameter family of binary populations. The details of this analysis will be reported elsewhere. In summary, an Abt and Levy (1976) distribution of binaries is rejected at the 96 percent confidence level unless all binaries closer than about $1 \mathrm{AU}$ are removed from the sample, or alternatively the fraction of binaries is reduced from 0.54 to less than about 0.2 .

\section{REFERENCES}

Abt, H. A., and Levy, S. G. (1976). Astrophys. J. Suppl. 30, 273.

Gunn, J. E., and Griffin, R. F. (1979). Astron. J. 84, 752.

Harris, H. C., and McClure, R. D. (1983). Astrophys. J. Lett. 265, L77.

Mayor, M., Imbert, M., Andersen, J., Ardeberg, A., Benz, W., Lindgren, H., Martin, N., Maurice, E., Nordström, B., and Prévot, L. (1984). Astron. Astrophys. $134,118$.

Tonry, J., and Davis, M. (1979). Astron. J. 84, 1511. 